\title{
Narrativa
}

Nuova serie

$41 \mid 2019$

Narrativa italiana degli anni Duemila: cartografie e percorsi

\section{La letteratura italiana all'epoca della crisi climatica}

\section{Chiara Mengozzi}

\section{(2) OpenEdition}

\section{Journals}

\section{Edizione digitale}

URL: https://journals.openedition.org/narrativa/346

DOI: $10.4000 /$ narrativa.346

ISSN: 2804-1224

\section{Editore}

Presses universitaires de Paris Nanterre

\section{Edizione cartacea}

Data di pubblicazione: 1 décembre 2019

Paginazione: 23-39

ISBN: 978-2-84016-350-3

ISSN: $1166-3243$

Notizia bibliografica digitale

Chiara Mengozzi, «La letteratura italiana all'epoca della crisi climatica», Narrativa [Online], 41 | 2019, online dal 01 novembre 2021, consultato il 08 décembre 2021. URL: http://journals.openedition.org/ narrativa/346 ; DOI: https://doi.org/10.4000/narrativa.346

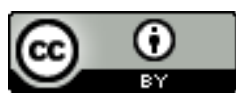

Narrativa est mise à disposition selon les termes de la Licence Creative Commons Attribution 4.0 International. 


\section{La letteratura italiana all'epoca della crisi climatica ${ }^{1}$}

\section{RiASSUNTO}

L'articolo esplora le diverse maniere in cui gli scrittori italiani contemporanei mettono in scena cause ed effetti (sociali, ambientali e psicologici) della crisi climatica in corso, offrendoci al contempo la possibilità di cambiare sguardo sul nostro Umwelt, inteso come spazio di coesistenza - fatto di incontri e simbiosi o, viceversa, di rapporti conflittuali e violenti - tra agenti differenti, umani e non-umani.

\section{RÉSUMÉ}

L'article montre la manière dont les écrivains italiens contemporains mettent en scène la crise climatique actuelle, avec ses causes et ses effets, qu'ils soient sociaux, environnementaux ou psychologiques. Leurs œuvres nous offrent la possibilité de changer de regard sur notre environnement, considéré comme un espace de coexistence - fait de rencontres et symbioses ou, vice-versa, de rapports conflictuels et violents - entre agents différents, humains et non humains.

\section{UNA QUESTIONE DI SGUARDO}

In seno alla comunità scientifica c'è oggi un largo consenso riguardo alla natura antropogenica dei cambiamenti climatici in corso. Secondo la tesi dell'Antropocene ${ }^{2}$, siamo ormai entrati in una nuova epoca geologica caratterizzata dagli

1. Il presente studio è stato condotto nell'ambito del progetto europeo KREAS "Creativity and Adaptability as Conditions for the Success of Europe in an Interrelated World" (No. CZ.02.1.01/0.0/0.0/16_019/0000734).

2. Introdotta da Paul Crutzen e Eugene Stoermer nel 2000, la nozione di Antropocene è al centro di un dibattito che concerne in particolare: 1) le prove stratigrafiche, 2) la datazione (alcuni, come William Ruddiman o Elizabeth Kolbert, propendono per far 
esseri umani come principale agente di trasformazione ambientale. La nostra azione sul pianeta, a partire dalla rivoluzione industriale e con una forte accelerazione dopo la seconda guerra mondiale, avrebbe conosciuto un salto quantitativo e qualitativo tale da poter mettere in pericolo, se non la vita in generale - che, su scale temporali lunghissime, ha sempre dimostrato una straordinaria resistenza - perlomeno la nostra sopravvivenza in quanto specie. Probabilmente mai saremo in grado di calcolare con precisione l'insieme delle retroazioni e delle reazioni a catena che l'aumento della temperatura potrebbe scatenare, ma proprio per questo, consigliano gli scienziati, è meglio adottare il principio di precauzione - se non di maximin - e cominciare ad agire subito: "If we want to solve the climate problem, we must phase out coal emissions. Period"3. Le cause, dunque, sono per gran parte note e così pure le misure da adottare, almeno in linea generale: ridurre drasticamente le emissioni di gas serra, l'impatto sulla biosfera, la pressione sulle altre specie viventi e gli ecosistemi, ecc. Eppure si stentano a prendere decisioni risolutive su scala globale. Perché il capitalismo è troppo pervasivo per essere smantellato; perché non siamo disposti a rinunciare al nostro stile di vita, tanto più sapendo che l'azione dei singoli sarebbe irrilevante se non si accompagna a misure strutturali; perché il riscaldamento climatico pone un problema di responsabilità differenziate (non tutti i paesi inquinano allo stesso modo) e di giustizia internazionale (il Sud del mondo non ha forse il diritto di seguire il modello di sviluppo occidentale?), ecc. Le difficoltà che ostacolano o ritardano l'azione, tuttavia, potrebbero anche essere di altra natura. Se nemmeno il rischio concreto di un pianeta che si appresta a diventare sempre più invivibile per tutti gli esseri umani (anche se in un primo tempo la crisi ambientale non farà che aggravare le differenze sociali) sembra sufficiente, c'è forse in gioco anche qualcos'altro: c'è un problema di sguardo, di visione, di immaginazione.

Ed è qui che la letteratura entra in campo. Di fronte alla magnitudine del problema e alla complessità della sua risoluzione, saremmo portati a pensare che il contributo offerto dal discorso letterario sia marginale e tutto sommato trascurabile. Ma scienziati, filosofi e politologi non la pensano così e, anzi, sono pronti a scommettere sulla sua utilità. La letteratura è mobilitata come

coincidere l'inizio dell'Antropocene con la rivoluzione neolitica, altri, come Paul Crutzen e Will Steffen, con l'invenzione del motore a vapore) e, infine, 3) l'uso della categoria di specie nel neologismo, visto che le società industrializzate sono più responsabili di altre della crisi in corso.

3. Hansen James, Storms of My Grandchildren: The Truth about the Coming Climate Catastrophe and Our Last Chance to Save Humanity, New York, Bloomsbury, 2009, p. 176. Cfr. Sunstein Cass, Worst-Case Scenarios, Cambridge-London, Oxford University Press, 2007. 
risorsa retorica e narrativa capace di rendere accessibili le previsioni degli scienziati; perché, contrariamente a calcoli, grafici e statistiche, ha il vantaggio di parlare alle emozioni dei lettori e perché può offrire delle visioni del futuro che ci fanno percepire che cosa potrebbe voler dire vivere sulla terra una volta che il nostro habitat si sarà trasformato al punto da diventare irriconoscibile. Si pensi alle metafore impiegate dagli scienziati per spiegare l'imprevedibile risposta del pianeta alle alterazioni causate dall'uomo ${ }^{4}$, oppure all'uso frequente dei what if scenario nelle opere di divulgazione scientifica ${ }^{5}$, o ancora all'interesse dimostrato di recente dagli studiosi di scienze politiche per le speculazioni ecologiche della fantascienza ${ }^{6}$.

È giocoforza constatare, tuttavia, che queste prospettive, proprio mentre attribuiscono un ruolo indispensabile alla letteratura, finiscono per confinarla a una posizione ancillare, riducendola alla sua immediata utilità, a strumento in grado di veicolare dati scientifici o di propagare una causa, per quanto giusta essa sia. C’è chi, però, si spinge oltre ed è disposto a scommettere piuttosto sulla portata cognitiva intrinseca delle opere letterarie, o artistiche in generale, se non altro perché queste ultime - anche qualora non trattino direttamente la crisi climatica o la mettano in scena in maniera obliqua - ci costringono comunque, attraverso l'esperienza del bello, o dell'Unheimliche, a ridimensionare il nostro "io", ad ammettere che la realtà circostante ci plasma almeno tanto quanto noi plasmiamo lei ${ }^{7}$. Questa esperienza di decentramento - come vedremo nelle pagine seguenti attraverso alcune opere della letteratura italiana recente - è tanto più forte quando a entrare in scena nella pagina letteraria è il cambiamento climatico, le cui manifestazioni ci obbligano a riconoscere non soltanto che la storia del pianeta non può e non deve essere raccontata esclusivamente dal punto di vista umano, ma anche che una prospettiva radicalmente antropocentrica è in fin dei conti intenibile e nociva innanzitutto e soprattutto per gli esseri umani: "the fact that this planet has life and processes that support life - scrive Chakrabarty - only forces on us the

4. Broecker Wallace, Kunzig Robert, Fixing Climate: What Past Climate Changes Reveal about the Currnt Threat - and How to Counter It, New York, Hill and Wang, 2008, p. 100: "Every now and then [...] nature has decided to give a good swift kick to the climate beast. And the beast has responded, as beasts will - violently and a little unpredictably".

5. Un esempio per tutti, il famosissimo libro di WeIsman Alan, The World Without Us, New York, Thomas Dunne, 2007.

6. Cfr. Rumpala Yannick, Hors des décombres du monde. Écologie, science-fiction et éthique du futur, Ceyzérieu, Champ Vallon, 2018.

7. È quanto afferma MorTon Timothy, Noi, esseri ecologici, Roma-Bari, Laterza, 2018. 
recognition that however we strategize, the planet remains a coactor in the processes that will delay or hasten climatic shifts"

Si diceva che la crisi climatica pone un problema di sguardo. Ebbene, l'accumulo di dati ansiogeni sulle percentuali di gas serra, sull'alterazione del ciclo del carbonio, sul crollo rovinoso della biodiversità, sullo scioglimento dei ghiacciai ecc. (in breve, quello che Timothy Morton chiama "la modalità discarica di informazioni'), non può che rimanere lettera morta se non è supportato da un profondo ripensamento del nostro stare al mondo in quanto specie homo sapiens; un mondo che - è bene rendersene conto e trarne tutte le dovute conseguenze - è composto quasi interamente da non-noi (animali, piante, batteri e microbi - soltanto il corpo di un uomo adulto ne contiene 100 bilioni -, per non parlare del mondo inanimato), e dove la frontiera tra noi e non-noi è sempre più porosa: gli effetti sconvolgenti del cambiamento climatico, in quanto non unicamente imputabili alla natura, si manifestano a noi in una forma d'inquietante familiarità e testimoniano di un collasso in corso delle frontiere tra storia umana e storia naturale.

C'è dunque una ragione cognitiva per esplorare il modo in cui gli scrittori italiani mettono in scena cause, effetti (sociali, ambientali e psicologici) e possibili "soluzioni" della crisi ambientale, in un paese, peraltro, particolarmente colpito dagli effetti dei cambiamenti climatici, nonché devastato dall'incuria ambientale, dall'abusivismo edilizio, dai rifiuti'. La letteratura ci offre, infatti, la possibilità di cambiare sguardo sul nostro Umwelt (inteso come spazio di coesistenza fatto di incontri e simbiosi o, viceversa, di rapporti conflittuali e violenti, tra agenti differenti, umani e non-umani) e, ancora più in generale, di riportare in superficie ciò che la modernità occidentale ha voluto rimuovere in nome del progresso, per esempio un'idea complessa del pianeta che abitiamo, il quale non rappresenta semplicemente un fondale inerte che ci permette di dispiegare a oltranza la nostra azione trasformatrice, né un qualcosa che possa essere interamente misurato, domato e sottomesso al calcolo delle probabilità.

8. Chakrabarty Dipesh, "The Human Condition in the Anthropocene", The Tanner Lectures on Human Values, Yale University, p. 183.

9. Si ispirano all'approccio ecocritico ormai numerosissimi articoli e volumi. Ci limitiamo qui a segnalare, per quanto riguarda l'Italia, i lavori pionieristici di Serenella Iovino (Ecologia letteraria: una strategia di sopravvivenza, Milano, Ed. Ambiente, 2006; EAD, Ecocriticism and Italy: Ecology, Resistance and Liberation, London, Bloomsbury Academics, 2016) e la ricchissima monografia di Niccolò SCAFFAI (Letteratura e ecologia, Roma, Carocci, 2017) che oltre ad avere il pregio di soffermarsi sugli aspetti retorici e formali dei testi, spesso trascurati in questo ambito di studi, presenta un capitolo dedicato interamente alla letteratura italiana. 
C'è, però, anche un'altra ragione per esplorare il nesso tra letteratura italiana e crisi climatica. Il cambiamento climatico - in quanto effetto più vistoso, insieme a quella che i biologi chiamano "sesta estinzione di massa", dell'epoca che va sotto il nome di Antropocene - apre, per letteratura in generale, e per quella italiana in particolare, un nuovo orizzonte di possibilità espressive: quali sono le sfide che questo "iper-oggetto" ${ }^{10}$ pone ai regimi di rappresentazione?" Come metterlo in scena o farne oggetto di racconto e riflessione? Attraverso quali codici, strutture, procedimenti e quali forme di intersezione con la scienza? E qual è "l'inconscio ambientale" delle opere? ${ }^{12}$ Come si manifestano, traducono, sedimentano nelle strategie retoriche dei testi le ansie del nostro presente oppure la rinnovata percezione della storia umana nei suoi rapporti di determinazione, conflitto e interdipendenza con quella naturale?

\section{Misurare LA REALTÀ CON LA PIANTA DEI PIEDI}

Tra gli autori italiani contemporanei che attribuiscono alla propria scrittura una marcata militanza ecologica e un impegno esplicito verso la crisi climatica, si possono annoverare Bruno Arpaia, Franco Arminio e Wu Ming 1. Si dirà che questi scrittori non hanno niente o quasi in comune, ma l'accostamento, in realtà, non è poi così peregrino. I loro progetti narrativi sono accomunati, innanzitutto, da una tendenza a incrociare il discorso letterario con altri saperi o sfere dell'esperienza umana, e, in secondo luogo, dal viaggio (a piedi) come tema e/o modalità conoscitiva. Qualcosa, là fuori di Arpaia è un romanzo di anticipazione caratterizzato da una particolare forma di realismo, il realismo speculativo, che spesso informa la fantascienza e così pure uno dei suoi più recenti sottogeneri che va sotto il nome di cli-fi, ancora poco praticato in Italia ${ }^{13}$.

10. Morton Timothy, Hyperobjects. Philosophy and Ecology after the End of the World, University of Minnesota Press, 2013.

11. Secondo Amitav Ghosh (The Great Derangement. Climate Change and the Unthinkable, Chicago-London, The University of Chicago Press, 2016; trad. it. La grande cecità. Il cambiamento climatico e l'impensabile, Vicenza, Neri Pozza, 2017), il romanzo realista occidentale avrebbe difficoltà a mettere in scena la crisi climatica perché esso relega l'improbabile e il meraviglioso in secondo piano per concentrarsi sul quotidiano e la regolarità della vita borghese, escludendo al contempo l'agency di "attori" non umani.

12. Parla di inconscio ambientale, ispirandosi al concetto di inconscio politico di Jameson, Lawrence BuELL nel suo libro Writing for an Endangered World: Literature, Culture, and Environment in the U.S. and Beyond, Cambridge, Harvard University Press, 2001.

13. L'etichetta, introdotta nel 2007 dal giornalista Dan Bloom, comprende anche i meteo thriller. Cfr. in Italia Montrucchio Alessandra, E poi la sete, Milano, Ambiente, 2011, e Grosso Alessio, Apocalisse bianca, Milano, Mursia, 2004. Sui numerosi 
La struttura portante del testo - ovverosia l'alternanza tra due piani temporali, rispettivamente il primo e l'ultimo quarto del nostro secolo - fornisce la possibilità, da un lato, di mostrare il decorso dei cambiamenti climatici insieme alle loro più vistose conseguenze socio e geopolitiche, dall'altro, di intrattenere due diversi tipi di dialogo con la scienza. Se nelle parti temporalmente più vicine a noi, quelle in cui la catastrofe potrebbe essere ancora scongiurata, gli attuali dibattiti scientifici sono drammatizzati nelle conversazioni che oppongono i diversi personaggi (tra gli altri, quello estremamente controverso sulle possibili "soluzioni" geo-ingegneristiche alla crisi climatica, come l'idea di far calare le temperature non riducendo ma addirittura incrementando l'impatto umano e tecnologico, per esempio iniettando nell'atmosfera molecole di acido solforico); nelle parti ambientate alla fine del secolo, invece, i dati forniti dalle previsioni scientifiche ${ }^{14}$ si traducono in lunghe descrizioni del paesaggio europeo che, dal nord Italia, passando per la Svizzera e la Germania, su fino alla Scandinavia, non ha più nulla di familiare. I passaggi più efficaci sono probabilmente proprio quelli in cui - seguendo l'estenuante tragitto a piedi compiuto dal protagonista Livio insieme a un convoglio di migliaia di profughi in fuga dall'Europa mediterranea - sfilano, sotto gli occhi dei personaggi e dei lettori, villaggi abbandonati, fiumi in secca, laghi trasformati in acquitrini, città inghiottite dal mare, germogli di palme laddove c'erano abeti, polvere e terra arida dove c'erano pianure verdeggianti. In questo futuro prossimo venturo, $i$ profughi che camminano disperati "senza vedere un metro di futuro davanti a sé"15 siamo proprio noi europei del Sud, la carta geopolitica del globo si è completamente ridisegnata (cittadine sperdute dell'estremo Nord come Iqaluit, Tromsø, Nuuk sono diventate il centro del mondo), mentre i luoghi dell'immaginario bucolico o agreste sono ormai irriconoscibili, malsani, ostili e non resta altro che adattarsi a questo nuovo Umwelt, oppure cercare

esempi anglosassoni, esistono anche alcune monografie. Tra le altre, Texter Adam, Anthropocene Fictions. The Novel in a Time of Climate Change, Charlottesville, University of Virginia Press, 2015.

14. Arpaia mira all'attendibilità. Da qui l'uso delle fonti scientifiche, opportunamente segnalate nell'apparato bibliografico, come è consuetudine nei romanzi appartenenti al genere. Per amore del vero, tuttavia, è bene segnalare che gli esperti (ingegneri, climatologici, biologi, ecc.) che animano il sito/blog www.climalteranti.it, hanno messo alla prova la sostanza scientifica del testo, sostenendo che gli scenari del romanzo andrebbero post-datati. Qui la recensione: https://www.climalteranti.it/2016/06/06/ il-romanzo-sugli-impatti-e-la-scienza-del-clima/.

15. Bruno Arpaia, Qualcosa, là fuori, Milano, Guanda, 2016, p. 88. 
disperatamente di entrare nei confini dell'Unione Europea del Nord, che però protegge militarmente le proprie frontiere.

Nel lavoro di Arminio e Wu Ming 1, il viaggio o, meglio, l'attraversamento a piedi del territorio, non è più soltanto un nucleo tematico fondamentale ma anche e soprattutto una pratica che accompagna e sostiene la scrittura, se non un vero e proprio metodo di indagine ${ }^{16}$. All'incrocio tra etnologia, geografia e poesia, la paesologia di Franco Arminio si nutre di un rapporto metaforico, metonimico e simbiotico tra il corpo (malato) dello scrittore e il paesaggio. Metaforico, perché la sua salute precaria (reale o immaginata, poco importa) rinvia continuamente all'agonia dei paesi irpini; metonimico perché "la ricognizione dei luoghi è il frutto di uno spostamento d'attenzione, dal sintomo del corpo al sintomo del luogo, dall'ipocondria alla desolazione" ${ }^{17}$; e infine simbiotico, cioè fatto di sconfinamenti reciproci, perché la paesologia è definita come "ill passare del mio corpo nel paesaggio e il passare del paesaggio nel mio corpo"18. Quest'ultimo tipo di rapporto è il più interessante perché in esso confluiscono l'aspetto programmatico e poetico della sua scrittura. Se il pianeta oggi è malato e il clima nervoso (le due immagini ritornano spesso) è perché, incapaci di pensare alla natura diversamente che come a una risorsa o a una discarica infinite, abbiamo dimenticato che "non siamo padroni del mondo", ma al contrario "siamo natura $[\ldots]$ animali nell'aria e stiamo insieme al muso dei conigli e agli aghi di pino, ci sono le nostre rughe e gli anelli degli alberi, ognuno sulle sue cellule raccoglie firme del tempo che passa"19. Ecco allora le numerose "prescrizioni", alle volte addensate in interminabili liste, talaltre disseminate nel tessuto narrativo scandito dalle tappe del percorso: imparare a potare un albero e a fare un caciocavallo, stare all'aria aperta almeno due ore al giorno, piantare alberi lungo le tangenziali, "prendere la propria anima e portarla lontano, dove non passano esseri umani: gli abissi marini, le vette più impervie"20, "pensare alle volpi morte sotto la neve, alle fatiche delle formiche, al verde lucidato dal vento" ${ }^{21}$. Si tratta di consigli che sono volti a soppiantare un rapporto strumentale agli spazi con uno di intensità

16. Si potrebbe aggiungere a questi esempi anche il racconto della ricerca compiuta da Simona Baldanzi seguendo il corso dell'Arno, in Maldifiume. Acqua, passi e gente dell'Arno, Portogruaro, Ediciclo, 2016.

17. Arminio Franco, Geografia commossa dell'Italia interna, Milano, Mondadori, 2013, p. 3.

18. Arminio Franco, Terracarne. Viaggio nei paesi invisibili e nei paesi giganti del Sud Italia, Milano, Mondadori, p. 11.

19. Arminio Franco, "Curiamo la natura o l'allerta meteo non servirà mai a nulla", Corriere della Sera, 2 novembre 2018.

20. ID., Geografia commossa dell'Italia interna, cit., p. 104.

21. Ibid., p. 7. 
poetica e sensoriale, di simbiosi originaria, di cura e simpatia, e che si materializzano in una scrittura che tende al decentramento dell'umano, a "seppellire la nostra presunzione di specie"22: per esempio quando l'autore ci invita ad adottare lo "sguardo del cane" ${ }^{\text {"23 }}$, vale a dire orizzontale e olfattivo, oppure quando ci fa vedere presenze e pienezza (di vita animale, vegetale, minerale) laddove saremmo portati a dire, di fronte a villaggi semi-abbandonati o in rovina, che si tratta di paesi ormai morti ("Craco accoglie il sole e la pioggia, accoglie il vento, l'alba e il tramonto. È ospitale con le rondini e con le mosche, è una dimora ideale per le capre e le lucertole"24) o, ancora, quando ci offre stralci della sua biografia senza far ricorso a date ufficiali o ad altri avvenimenti umani: al momento della sua nascita, "era febbraio del sessanta, c'erano nel paese una decina di macchine e un migliaio di muli, le rondini muovevano il cielo, i porci tenevano ferma la terra, $\mathrm{i}$ giorni camminavano quieti verso il futuro" 25 .

"Noi siamo parte di questa onda di scrittori che negli ultimi anni ha cominciato a riflettere sulla viandanza come strumento conoscitivo"26, afferma Wu Ming 1 in riferimento non soltanto al "trittico alpino" 27 , ma anche all'ampio progetto in fieri di mappatura, attraversamento e racconto del Delta del Po, una delle aeree italiane più colpite dai cambiamenti climatici, che provocano, oltre alla siccità delle sorgenti e all'abbassamento del livello del fiume a valle, anche un'inversione del flusso dell'acqua salata dell'Adriatico, che risale il Po per svariati chilometri, con un impatto enorme sugli ecosistemi e l'agricoltura. Il progetto consiste nell'intraprendere un viaggio nello spazio e nel tempo per raccontare, all'incrocio tra la strada (mettersi in cammino per fotografare i luoghi e intervistare gli abitanti) e l'archivio (dove recuperare le tracce delle bonifiche nonché delle lotte sociali che le hanno accompagnate), questo territorio iper-ingegnerizzato, che è il risultato di 400 anni di drenaggi e risanamenti, ed è fatto di terre emerse che, dopo essere state faticosamente strappate all'acqua, rischiano di ritornare sommerse, come

\section{Ibid., p. 49.}

23. Cfr. Arminio Franco, Terracarne, cit., pp. 9-10.

24. ID., Geografia commossa dell'Italia interna, cit., p. 86.

25. Ibid., p. 117.

26. La visione dal basso. Una conversazione tra Wu Ming 1 e Italian Limes su clima, territorio, confini, esplorazioni, https://www.wumingfoundation.com/giap/2019/03/italian-limes/. È probabile che Wu Ming 1 si riferisca proprio a Franco Arminio e, ancor prima, a Gianni Celati. Sul forte legame tra questi due scrittori, vedi Iacoli Giulio, "L'invenzione della 'paesologia'. Franco Arminio e le nuove zone del racconto in Italia", Compar(a)ison, n. 1, 2008, pp. 78-80.

27. Cfr. Point Lenana (2013), Cent'anni a Nordest (2015) e Un viaggio che non promettiamo breve (2016). 
effetto involontario delle azioni umane sull'ambiente. All'origine di questo ambizioso progetto, che per il momento porta il titolo di Blues per le terre nuove, se ne situa un altro, al confine tra parole e musica: $A r z ̌ e ̀ s t u l a^{28}$ è infatti il titolo sia di un racconto di finzione firmato da Wu Ming 1, sia di un album composto dai Funambolique che del racconto è la colonna sonora ${ }^{29}$. In seguito a una crisi ecologica e politica non meglio precisata che ha riportato l'Italia a uno stato premoderno, causato lo spopolamento della penisola, instaurato un regime di sussistenza e un governo fantoccio quasi esclusivamente impegnato a respingere i profughi ecologici in fuga da altre aree del pianeta, una donna, che si fa chiamare la Scrittrice, la Storica o la Veggente, decide di mettersi in cammino per ritrovare i luoghi della sua infanzia nei pressi di Ferrara. In seguito a questo viaggio, farà ritorno all'ex-autogrill di Cantagallo, vicino a Bologna, dove vive con una strana comunità di reietti, insieme ai quali si dedica a pratiche visionarie del futuro, guardando in alternanza gli astri, che irradiano la luce di migliaia di anni fa, il pianeta e la loro terra, quella dove un tempo sorgeva Bologna ed ora c'è un grande bosco. Mentre attraversa le macerie degli insediamenti umani ora riconsegnati alla vita animale e vegetale, le ritornano in mente le parole del dialetto ferrarese scomparso (Argùr, Zarabìgul, Arzèstula - ramarro, formicaleone, cinciallegra) e, proprio mentre le nomina, la cinciallegra, "struggente meraviglia dell'evoluto" 30 , si materializza su un ramo: "prima degli enti mancarono le parole. E adesso che gli "enti" tornano, e chiurli ne sento spesso e le sere d'estate è pieno di lucciole, le parole sciorzz e baciosa sono più morte che mai"'31. Quello che in Blues per le terre nuove è ormai diventato un programma esplicito, nel racconto si affacciava in forma allegorica: cercare un avvenire nel passato, guardare insieme al futuro della nostra casa terrestre e immaginarlo scavando innanzitutto nella memoria dei luoghi, tracciando e seguendo, passo a passo, le loro trasformazioni.

28. Wu Ming 1, Arzèstula, in Vasta Giorgio (a cura di), Anteprima nazionale. Nove visioni del nostro futuro invisibile, Roma, Minimum Fax, 2009, pp. 83-105.

29. Per Wu Ming 1 si tratta di sintonizzarsi con il territorio del delta del Po attraverso il maggior numero possibile di mezzi espressivi. Vengono in mente, a questo proposito, le riflessioni di Amitav Ghosh nel già citato The Great Derangement, dove, ispirandosi a Edouard Kohn (Comment pensent les forêts), si chiede se il linguaggio verbale sia il mezzo migliore per pensare il pianeta all'epoca dei cambiamenti climatici, e se non sia invece più efficace pensare per immagini (o per suoni, potremmo aggiungere) inventando nuove forme ibride.

30. Wu Ming 1, Aržèstula, cit., p. 97.

31. Ibid., p. 87. 


\section{LA FINE DELL'ANTROPOCENTRISMO TRANQUILLO}

Attinge a piene mani all'immaginario catastrofico, distopico e (post)apocalittico, oltre a Wu Ming 1, anche la gran parte degli scrittori italiani che in questi ultimi anni si sono dimostrati ricettivi nei confronti della questione climatica senza per questo investire la propria scrittura di una dichiarata e programmatica missione ecologica. L'(ab)uso del paradigma apocalittico nella mise en récit dei problemi ambientali è stato comprensibilmente criticato da più fronti. Continuare a rappresentarci nell'imminenza di una catastrofe inevitabile sarebbe, per qualcuno, semplicemente deresponsabilizzante e controproducente ("c'est maintenant ou jamais qu'on peut jouir de façon paisiblement destructrice des ultimes beautés du monde" 32 ), per altri, una vera e propria strategia di rimozione, con effetti piacevoli e rassicuranti: come nei sogni da sindrome post-traumatica, staremmo cercando di collocarci prima del trauma nel tentativo disperato di poterlo anticipare ed evitare ${ }^{33}$. Ma la catastrofe climatica, lungi dall'essere un non-ancora, è già cominciata, anche se non siamo stati in grado di vederlo e stentiamo ancora a prenderne atto, perché, come per tutti gli eventi traumatici, "ciò che sta per piombarti addosso si trova sempre nel punto cieco dello specchietto retrovisore" "34. Non c'è dubbio che il rischio di una produzione letteraria e cinematografica che indugia a oltranza nell'estetizzazione della catastrofe, inquadrandola in scenari e intrighi stereotipici, sia quello di produrre un effetto derealizzante e di assuefazione. Non è tuttavia necessariamente così. Se è vero che è proprio nel momento in cui un meccanismo si inceppa o qualcosa si rompe che l'oggetto, sottratto all'automatismo del suo utilizzo, si manifesta sotto una nuova luce, allora le narrazioni della fine o della catastrofe - parola che, secondo l'etimologia greca, significa capovolgimento - sono anche quelle che mostrano fino a che punto le coordinate e i paradigmi con cui abbiamo

32. Cfr. Castel Pierre-Henri, Le Mal qui vient. Essai hâtif sur la fin des temps, Paris, Éditions du Cerf, 2018, p. 61.

33. Cfr. Morton Timothy, Noi esseri ecologici, cit.

34. Scurati Antonio, La seconda mezzanotte, Milano, Bompiani, 2012, p. 342. L'idea è ripresa, con alcune varianti, in diversi punti del romanzo e compare persino nell'incipit: "Alla fine la sera occidentale era arrivata, ma gradualmente, e noi non ce n'eravamo nemmeno accorti" (p. 11). Ritorna, inoltre, in Cinacittà di Tommaso Pincio ("Prima poco a poco, poi all'improvviso", così afferma più volte il narratore protagonista, riferendosi certo alla propria sciagurata vicenda, ma indirettamente anche al "famoso anno senza inverno", segno di un disastro climatico ormai consumato), dove - a detta di molte recensioni - il riscaldamento climatico sarebbe soltanto un pretesto. $\mathrm{Ma}$ in un contesto di semiosi illimitata come il discorso letterario l'ambientazione può essere davvero un dettaglio irrilevante e interscambiabile? 
interpretato il nostro stare al mondo non funzionino più, perché sono esattamente quelli che hanno portato, o porteranno a breve, alla rottura degli equilibri planetari. Come ad esempio accade nei romanzi di Antonio Scurati, Laura Pugno e Francesco Pecoraro ${ }^{35}$, i quali testimoniano, attraverso delle precise scelte tematiche e formali, di come oggi, nel bel mezzo di una crisi climatica e ambientale, "non possiamo più godere di un antropocentrismo tranquillo"36, non possiamo più ignorare che la frontiera tra l'umano e il non-umano, inteso come confine interspecifico ma anche, più in generale, come confine tra storia umana e naturale, che pensavamo risolto tutto a vantaggio del primo termine, stia invece precipitosamente collassando.

La seconda mezzanotte di Scurati è un vero e proprio metaromanzo della catastrofe, nella misura in cui uno dei motivi centrali del testo è proprio la messa in scena del consumo televisivo, perverso, compiaciuto e impotente di esperienze estreme: disastri ambientali e guerre in primis. In un mondo dove il riscaldamento climatico ha devastato ampie zone del pianeta, travolgendo la gran parte degli apparati statali, Venezia - quasi interamente inghiottita da un'Onda che ha portato gli abitanti a dimenticare progressivamente il mondo com'era prima - si trova nelle mani di una multinazionale cinese che l'ha in parte ricostruita, sollevando un grande muro di cinta intorno alle parti salvate e un superdome climatizzato sopra Piazza San Marco. Mentre il mondo là fuori è pura lotta per la sopravvivenza, all'interno si vive, e si gode, da spettatori di atrocità di ogni sorta: televisive, innanzitutto, perché trasmesse su megaschermi dai cinedisastri che chiosano con "Enjoy the inner world", oppure offerte ai turisti metropolitani sotto forma di cruenti combattimenti tra gladiatori nell'arena di Piazza San Marco. La Venezia "salvata" è un bordello della fine dei tempi dove spettacolo e consumo sfrenato vanno a braccetto, dove ai pochi privilegiati è elargito il puro godimento della distruzione ultima, accelerata, sfrenata di ogni risorsa restante: tra un massacro di gladiatori e l'altro, come in un'orgia parossistica, si alternano smisurati abusi sessuali, sanguinolente carneficine di animali, interminabili pasti pantagruelici a base di carne (c'è persino chi si fa impiantare dei bypass bilio-pancreatici per poter mangiare senza sosta). L'altra Venezia è quella semisommersa, la Venezia cosiddetta perduta. In realtà, questa Venezia perduta,

35. Meriterebbero un'analisi in questo percorso anche Vassalli Sebastiano, Dio il diavolo la mosca nel grande caldo dei prossimi mille anni, Torino, Einaudi, 2008; PINCIO Tommaso, Cinacittà, Torino, Einaudi, 2008, e ZаnotTi Paolo, Bambini bonsai, Milano, Ponte alle grazie, 2010.

36. Intervista a Laura Pugno in MACiLENTI Alessandro, Characterizing the Anthropocene. Ecological Degradation in Italian Twenty-First Century Writing, Berne, Peter Lang, 2018, p. 183. 
di cui i giovani conoscono soltanto le dicerie dei vecchi, è anche una città ritrovata per quelle forme di vita - mosche, topi, cani, gabbiani, rovi, gerani, ecc. che tenacemente sopravvivono riappropriandosi di quegli spazi abbandonati dagli uomini. Quello che mi sembra interessante, e che nelle recensioni del romanzo non mi pare emerga, è che la ribellione di Spartaco, in un primo momento esclusivamente individuale, si trasforma nella volontà di un'azione collettiva, proprio dopo aver attraversato il muro e squarciato simbolicamente il cielo finto del superdome. Dall'altra parte del muro, non soltanto tocca con mano gli effetti della devastazione causata dall'uomo, ma si trova anche di fronte a una piccola comunità di sopravvissuti che coltiva le terre riemerse dopo l'Onda e a quella tenace natura animale e vegetale, tanto ostile quanto stupefacente, che, mentre nella Venezia "salvata" è soltanto materia da masticare, digerire e defecare, qui continua a vivere malgrado e oltre l'uomo: ammaliato dallo straordinario rigoglio delle vigne selvatiche sospese sui tetti, in ascolto dei canti d'amore delle rane, incantato dalla bellezza dei cani che pur sono pronti a sbranarlo, Spartaco "entra in risonanza" ${ }^{37}$ persino con l" "eco di cose ignote e perdute", per esempio "con il destino mondiale dei pesci, le creature boccheggianti degli abissi [...] quasi percependo il rantolo salire all'esofago delle tartarughe marine ingolfato da sacchetti di plastica scambiati per meduse fosforescenti" ${ }^{\prime 38}$. Il giovane gladiatore farà dunque ritorno dai suoi compagni - anche perché fatto prigioniero dagli uomini del governatore - ma, invece di ritentare la sorte in un'altra fuga solitaria, capisce che, se una qualche forma di salvezza e di futuro esiste ancora, questa risiederà piuttosto in un'azione di gruppo. Per questo si metterà a capo di una rivoluzione il cui esito, benché compromesso dal probabile tradimento del Maestro, rimane comunque aperto, come a dischiudere una "tenue ma combattiva speranza" 39 . In un universo narrativo così opprimente e disperato (ma del resto viviamo in un mondo dove l'Earth Overshoot Day retrocede precipitosamente ogni anno), non è poco.

Claustrofobico e quasi senza vie di scampo anche l'universo narrativo del primo romanzo di Laura Pugno, Sirene; un'opera che, analogamente a quella di Scurati, si permette di regalarci unicamente nella soglia estrema del testo un momento di sollievo, per quanto paradossale perché orientato al superamento del confine di specie: al lettore è concesso infatti un estemporaneo fremito di

37. Scurati Antonio, La seconda mezzanotte, cit., p. 252.

38. Ibid.

39. Matarazzo Pierfrancesco, "Dialogo con Antonio Scurati sul suo ultimo romanzo La seconda mezzanotte", Sul Romanzo, 14 dicembre 2011, http:/ /www.sulromanzo.it/blog/ dialogo-con-antonio-scurati-sul-suo-ultimo-romanzo-la-seconda-mezzanotte. 
piacere soltanto quando la sirena mezzoumana può finalmente abbandonarsi "alla potenza della corrente, alla sua gioia decisiva" ${ }^{40}$, grazie al solo vero gesto di generosità del protagonista Samuel, che consiste nel liberarla e offrirle in pasto il proprio corpo come nutrimento, investendo in tal modo di significato la propria morte. Come scrive l'autrice, si tratta di "un fatto non triviale in un'apocalissi. In circostanze disperate, la conoscenza tecnica di cui dispone [vale a dire il funzionamento delle vasche di allevamento] gli permette, se non di salvare se stesso, di salvare comunque qualcosa" ${ }^{\prime 4}$. Non è mai spiegato nel romanzo il rapporto tra la scoperta delle sirene e l'epidemia di cancro nero che sta condannando all'estinzione il genere umano, né si conoscono le ragioni della moria di sirene che immediatamente segue la loro scoperta. Questa assenza di legami espliciti di causalità risponde a una logica narrativa ben precisa: invece di cercare di comprendere o semplicemente riconoscere le cause dei disastri in corso, gli esseri umani si limitano a cercare delle soluzioni tampone (per esempio la costruzione di resort suboceanici dove i ricchi si proteggono temporaneamente contro la potenza devastatrice del sole, ma le risorse cominciano a scarseggiare e il cancro nero finirà per divorare anche loro), senza mai mettere in discussione il proprio atteggiamento predatorio che si spinge fino alla rifunzionalizzazione economica del disastro ambientale (i turisti accorrono numerosi al macabro spettacolo delle sirene morte sulla spiaggia). In realtà, non servono troppe spiegazioni al lettore per capire che il controllo su quello che chiamiamo natura, precisamente nel momento in cui si concepisce come illimitato (il mondo di Sirene è caratterizzato da uno straordinario sviluppo scientifico-tecnologico), si trasforma nel suo contrario, ovverosia in una serie di effetti non previsti e non intenzionali che neutralizzano questa pretesa (per esempio il cancro nero, in seguito alla trasformazione della composizione dell'atmosfera). Al culmine del progresso e dello sfruttamento capitalistico del pianeta, le sirene emergono dagli abissi come un rimosso, perché su questa ultima specie selvaggia, si polarizza, manifestandosi alla luce del sole, quella violenza che silenziosamente si sta consumando ogni giorno ai danni di altre specie viventi, o destinate all'estinzione, oppure confinate in riserve naturali di dimensioni sempre più ridotte, o ancora allevate intensivamente per essere spedite al macello, come tocca infatti alle sirene. Crimini che come tali non vogliono qualificarsi perché un animale non si uccide, lo si sopprime; un animale non muore, bensì cessa di esistere.

40. Pugno Laura, Sirene, Torino, Einaudi, 2007, p. 141.

41. Intervista a Laura Pugno in Macilenti Alessandro, Characterizing the Anthropocene, cit., p. 187. 
Ed è proprio affinché il comportamento degli uomini si manifesti in tutta la sua inumana brutalità, che le sirene di Laura Pugno non sono soltanto creature diverse e impenetrabili, ma anche pericolosamente prossime a noi, a tal punto che con esse ci si può accoppiare e avere anche dei figli. A creare un forte effetto disturbante contribuisce in maniera fondamentale la prosa di Laura Pugno che, come è stato giustamente detto, è "refrattaria all'enfasi [...] improntata a un tono gelido, che non ammette deroghe" 42 . Questo, unito a "una voce narrante monocorde, totalmente anti-empatica", crea quel senso di insopportabile claustrofobia di cui si parlava all'inizio. La conclusione del romanzo, con la sirena mezzoumana che, di nuovo libera nell'oceano, dà alla luce un altro esemplare femmina per tre quarti sirena e per un quarto umana, e ripete il nome di Samuel senza però ricordarne il senso, perché la sua mente "è tabula rasa" non è né utopica né distopica, ma semplicemente e radicalmente darwiniana. Così come tutte le specie sono in movimento, anche l'homo sapiens è una forma transitoria dell'evoluto destinata a un suo superamento, tanto più che le sirene, a differenza degli uomini, sono più adatte al nuovo ambiente perché immuni al cancro nero ${ }^{44}$.

Autenticamente darwiniano anche e soprattutto il penultimo romanzo di Francesco Pecoraro, La vita in tempo di pace: "Darwin - afferma l'autore in un'intervista - è stato il pensatore più importante per la mia modesta visione del mondo. [...] Sono convinto che dobbiamo sforzarci di vedere il mondo senza cedimenti attraverso la chiave del materialismo evoluzionista", vale a dire nei termini di un processo di evoluzione non lineare e senza fine "il cui scopo è

42. Matт Luigi, "Una gelida distopia: le Sirene di Laura Pugno”, Treccani Magazine, 26 luglio 2017, http://www.treccani.it/magazine/lingua_italiana/articoli/percorsi/ percorsi_126.html.

43. Pugno Laura, Sirene, cit., p. 145.

44. Affascinata dalla paleoantropologia, Laura Pugno ha scritto anche una pièce teatrale sulle metamorfosi dell'umano, DNAct (Civitella in Val di Chiana, Zona, 2008). Nella già citata intervista afferma: "Abbiamo sempre a che fare con un altro che non è umano e il nostro passato ci informa che anche in termini evolutivi è stato così. Abbiamo convissuto con diversi ominidi, altre specie umane leggermente diverse con cui a quanto pare, secondo gli ultimi studi, ci siamo anche incrociati - il che dimostra che l'evoluzione è tutt'altro che un procedere lineare da una forma scimmiesca ad una forma perfetta. È una storia molto più complessa. Dato che quando queste interazioni sono avvenute eravamo già degli esseri in grado di produrre cultura, da qualche parte c'è probabilmente una sorta di relitto mnestico con cui dobbiamo fare i conti, quindi l'Altro è da sempre la domanda che ci portiamo dietro, non importa se animale o umano", in Macilenti Alessandro, Characterizing the Anthropocene, cit., pp. 185-186. 
leggibile solo a posteriori o non è leggibile affatto" ${ }^{45}$. Anche per questa ragione, il romanzo presenta una struttura temporale a ritroso. Ne La vita in tempo di pace la catastrofe climatica ed ecologica è onnipresente: non c'è capitolo che non indugi sull'infinita lista di segnali che annunciano uno scenario ambientale apocalittico (la grande alluvione di Roma, la morte del mare, lo sbiancamento dei coralli, ecc.). Due vie, cinicamente realiste, restano praticabili: l'alternativa è quella tra il falso-vero (vale a dire la museificazione di quel poco che resta della natura, da trasformarsi in "paesaggi terrestri a tema") e tra il falso-falso (vale a dire la sostituzione della natura con una completamente artificiale, "ma talmente ben fatta da risultare più vera del vero, arci-vera, un'iper-natura turgida $\mathrm{e}$ feconda"46), che è poi quello che si piega a fare l'ingegner Ivo Brandani, impegnato a ricostruire con materiali sintetici la barriera corallina del Mar Rosso.

Il futuro, dunque, non si è soltanto deteriorato, ma si è anche drasticamente accorciato: "venti? Trent'anni? Cento anni? [...] Mi perderò l'apocalisse, anche se probabilmente sarà un evento a sfumare" 47 . Ecco un'altra ragione che spiega perché i capitoli che seguono la consueta direzione della freccia temporale racchiudono soltanto una manciata di ore, mentre quelli in senso inverso ripercorrono settant'anni di storia: perché il tempo che resta al pianeta così come l'abbiamo conosciuto e, parallelamente, quello che resta al protagonista (che ancora non lo sa, ma sta per morire a causa di una meningoencefalite purulenta causata dal Naegleria fowleri), probabilmente non è molto: Brandani come individuo, e noi come specie homo sapiens, dobbiamo forse rassegnarci all'idea di aver già compiuto la più lunga porzione del nostro passaggio sulla terra. Si dirà che nel romanzo di Pecoraro c'è anche molto altro, al di là delle questioni ecologiche, per esempio ci sono gli ultimi settant'anni di storia politica e culturale d'Italia; e infatti, la specifica struttura temporale del testo serve a tenere insieme anche una lunga (auto)biografia individuale e collettiva, ma al riparo dall'illusione biografica di cui parla Bourdieu, perché la vita di un individuo e di una nazione non costituiscono in alcun modo un insieme coerente e orientato, e se proprio vogliamo vederci una trama fatta di necessità, nessi causali e finalità, non possiamo che far opera di riflessione e di selezione e, quindi, raccontarla al contrario. Non c'è qui la pretesa di esaurire l'analisi di un romanzo che è stato definito come uno dei più importanti degli ultimi decenni e sul quale sono già

45. PAnichi Camilla, "Una lotta sottile di tutti contro tutti. Intervista a Francesco Pecoraro", Le parole e le cose, 7 maggio 2014, http://www.leparoleelecose.it/?p=14909.

46. Pecoraro Francesco, La vita in tempo di pace, Milano, Ponte alle Grazie, 2013, p. 146. 47. Ibid., p. 148. 
state scritte pagine convincenti ${ }^{48}$. Quello che mi preme segnalare in questo percorso, è la capacità che Pecoraro dimostra nel farsi carico di tutta la complessità di relazioni che intercorrono tra la storia umana e quella naturale e nel tenere insieme scale temporali molto diverse ${ }^{49}$ : quella lunghissima delle ere geologiche e della vita sul pianeta, di cui come specie bomo sapiens facciamo parte, e quella ormai secolare del capitalismo, ma che in Italia coincide per lo più con la grande accelerazione e, quindi, con l'arco di vita del protagonista: un uomo che, malgrado le sue elucubrazioni infastidite per lo stato attuale delle cose, sa di incarnare alla perfezione "la classe media uniforme dell'Occidente democratico", proprio quella che si è succhiata il pianeta fino all'osso. Come ha giustamente sottolineato Dipesh Chakrabarty, la crisi climatica è la dimostrazione che "the time of human history [...] has now collided with the timescales of two other histories, both deep time, the time of the evolution of life on the planet, and geological time" ${ }^{50}$. Dunque, per comprendere quello che sta accadendo (ma anche per poter sperare di agire in qualche modo), è necessario zoomare avanti e indietro tra diverse scale e prospettive. A volte basta a Pecoraro un solo aggettivo per farci sprofondare in vertiginosi flash back: "fuori, lo scroscio di quella pioggia pleistocenica" ${ }^{\text {"51; }}$; più spesso è grazie a interminabili ma appassionanti digressioni che la storia umana dei luoghi che abitiamo si ricongiunge a quella immemoriale delle trasformazioni geologiche, per esempio quando il narratore, per descriverci quello che si trova fuori dalla porta a vetri della sua casa d'infanzia e quindi approfondire un tema portante dell'opera narrativa di Pecoraro, ovverosia il rapporto tra forma mentis \& forma urbis ${ }^{52}$, spalanca letteralmente la dimensione spazio-temporale del presente per sprofondare in un periodo compreso tra 630.000 e 360.000 anni fa, facendoci vedere le portentose colate laviche dei vulcani su cui si erge la Città di Dio, per poi giungere a 40.000 anni fa,

48. Per una lista dei principali studi sull'autore cfr. CoRTELLESSA Andrea (a cura di), La terra della prosa. Narratori italiani degli anni Zero (1999-2014), Roma, L’orma, 2014, p. 676.

49. Quanto scrive Carla BenedetTi a proposito di Gadda e Moresco in Disumane lettere. Indagini sulla cultura della nostra epoca (Roma-Bari, Laterza, 2011), e della loro capacità di proiettare i fatti politici e sociali "su quel più vasto orizzonte che è cosmico e biologico e sui tempi lunghissimi della storia umana sul pianeta" (posizione kindle 247/4346), mi pare vero anche per la prosa di Pecoraro. Questo è valido anche per le altre opere dell'autore, in particolare la raccolta Questa e altre preistorie e il recentissimo Lo stradone.

50. Chakrabarty Dipesh, "The Human Condition in the Anthropocene", cit., p. 179.

51. Pecoraro Francesco, La vita in tempo di pace, cit., p. 60.

52. Cfr. Bianchi Luisa, "La vita in tempo di pace di Francesco Pecoraro. Lo spazio come scontro di natura e cultura", in TuRI Nicola (a cura di), Ecosistemi letterari. Luoghi e paesaggi nella finzione novecentesca, Firenze, Firenze University Press, 2016, pp. 177-94. 
quando, sempre sullo stesso territorio, si è consumato il probabile sterminio dell'Homo neanderthalensis a causa dei sapiens, i nostri padri, quindi noi : "il resto - aggiunge il narratore - vogliamo considerarlo stranoto" 53 . Il procedimento dello straniamento non si applica soltanto ai luoghi, ma anche agli oggetti (di cui si traccia tutto il percorso dalla produzione, al consumo, alla loro trasformazione in rifiuti), o alle persone (e già l'uso frequente della definizione tassonomica bomo sapiens va in questa direzione), come nel passaggio, giustamente celebre, sullo sforzo del protagonista di vedere il piede della ragazza seduta accanto in aeroporto come "mano-di-primate-femmina-deformata-dalla-stazione-eretta" $"$.

In un'epoca di crisi climatica possiamo ancora raccontarci una storia esclusivamente intra-umana come se il pianeta si riducesse a noi? Come se "la-realtà-fuori-di-noi [fosse] un universo al nostro servizio" 55 ? Di fronte alla devastazione in corso, possiamo davvero continuare a guardare gli oggetti che produciamo e poi gettiamo come se la loro presenza si limitasse al solo frangente del loro utilizzo? Possiamo ancora far finta che il territorio che abitiamo, incidiamo, massacriamo non continui ad agire e retroagire sulla nostra storia?

$\grave{E}$ in tal modo, attraverso una struttura temporale inedita e grazie a cambi improvvisi di punti di vista e di scale, che nel romanzo di Pecoraro il senso della catastrofe diviene potenza di (re)visione: del pianeta e del nostro stare al mondo.

Chiara Mengozzi

Università Karlova, Praga

53. Pecoraro Francesco, La vita in tempo di pace, cit., p. 395.

54. Ibid., p. 144.

55. Ibid., p. 20. 
